\title{
ALGAZI, Gadi, Herrengewalt und Gewalt der Herren im späten Mittelalter. Herrschaft, Gegenseitigkeit und Sprachgebrauch
}

Joseph Morsel

\section{CpenEdition}

Édition électronique

URL : http://journals.openedition.org/ifha/1246

DOI : $10.4000 /$ ifha. 1246

ISSN : 2198-8943

\section{Éditeur}

IFRA - Institut franco-allemand (sciences historiques et sociales)

Référence électronique

Joseph Morsel, «ALGAZI, Gadi, Herrengewalt und Gewalt der Herren im späten Mittelalter. Herrschaft, Gegenseitigkeit und Sprachgebrauch », Revue de l'IFHA [En ligne], Date de recension, mis en ligne le 01 janvier 2001, consulté le 22 septembre 2020. URL : http://journals.openedition.org/ifha/1246 ; DOI https://doi.org/10.4000/ifha. 1246

Ce document a été généré automatiquement le 22 septembre 2020.

(C)IFHA 


\title{
ALGAZI, Gadi, Herrengewalt und Gewalt der Herren im späten Mittelalter. Herrschaft, Gegenseitigkeit und Sprachgebrauch
}

\author{
Joseph Morsel
}

1 La présence récurrente (sinon l'omniprésence) de la faide à la fin du Moyen Âge a déjà été constatée depuis longtemps, en particulier dans le quart sud-ouest de l'Empire mais pas uniquement comme l'ont montré ces dernières années des travaux sur la Westphalie, l'espace hanséatique ou les régions de Herford ou de Göttingen. De multiples schémas explicatifs ont été avancés, privilégiant selon les cas le problème des modalités de règlement des conflits (donc la conséquence d'un sous-développement juridique), la lutte contre la crise économique (donc la conséquence d'un sousdéveloppement économique), la genèse, sous une forme princière ou urbaine, de l'État moderne (donc la conséquence d'un sous-développement politique), voire la domination sur les dépendants paysans (donc la conséquence d'un sous-développement social). C'est notamment dans cette dernière perspective qu'Otto Brunner avait travaillé et acquis sa célébrité, en posant le caractère légal de l'usage de la force au Moyen Âge - ce qui en faisait une référence historique particulièrement utile à l'époque où il écrivait son ouvrage, Land und Herrschaft, en 1939 (qui en était déjà à la 3e édition en 1943 !).

2 C'est en particulier contre cette idée de la prétendue légalité médiévale du droit du plus fort que G.A. a repris l'examen de la pratique seigneuriale de la faide à la fin du Moyen Âge dans le Sud-Ouest de l'Allemagne, pour nous livrer un ouvrage passionnant en dépit des critiques que l'on peut émettre à son égard. L'ouvrage est en fait constitué de deux panneaux thématiques, bien sûr articulés l'un à l'autre, qui correspondent à des travaux en partie menés (voire publiés) séparément : le premier concerne l'examen des aspects langagiers et communicationnels de la domination seigneuriale (i.e. la domination seigneuriale se réalise aussi fondamentalement par le discours), examinés à 
travers une pratique extrêmement fréquente (mais peu ou mal étudiée) dans l'Empire, l'aveu des droits seigneuriaux (Weisung) par les habitants d'un village donné ; le second concerne spécifiquement le sens donné par des textes de type narratif ou idéologique à la pratique de la faide, constituée ainsi en enjeu d'entreprises offensives de catégorisation sociale. Le titre de l'ouvrage joue de ce fait sur les deux sens du mot Gewalt, notamment au Moyen Âge : à la fois « violence » (physique) et « autorité » (i.e. violence sociale).

3 L'étude de G.A. repose en premier lieu sur un remarquable exemple du caractère " déroutant " de l'analyse sémantique, qui ouvre des pistes de lecture jusqu'alors inconcevables. G.A. s'en prend ainsi à une notion centrale chez Brunner, le Schirm (" protection ") dû par les seigneurs à leurs tenanciers (que G.A. désigne systématiquement comme Bauern, "paysans ", contribuant ainsi à objectiver une catégorie sociale dont la formation et la définition sont pourtant un puissant enjeu à l'époque considérée - ce qu'il avait d'ailleurs lui-même envisagé dans O.G. Oexle (dir.), Memoria als Kultur, Göttingen, 1995) en contrepartie des redevances. L'examen de schirm et du verbe schirmen (dont il faut cependant reconnaître l'étroitesse typologique et numérique du corpus) fait apparaître un champ sémantique à la fois plus large et surtout plus négatif que ce que l'on pensait : la "protection » en question s'apparente à du " racket ", et ce sont moins les menaces extérieures au rapport seigneur-tenanciers que celles internes au rapport (l'alourdissement de la ponction) que les tenanciers espèrent conjurer par leurs versements. Exit la réciprocité enchantée...

4 Pourtant, la menace extérieure existe, sous la forme de faides récurrentes qui touchent au premier chef les tenanciers. Mais précisément, cette menace n'est que l'aspect complémentaire de la pression du seigneur sur ses dépendants : toute faide a comme conséquence la production chez les tenanciers d'un besoin d'être défendu, ce qui revient à dire, par exemple, que deux seigneurs voisins en guerre l'un contre l'autre produisent en fait chacun l'affermissement de la proximité seigneur/dépendants de l'autre. La domination seigneuriale se produit ainsi non par la soumission générale, militaire et visible de l'ensemble des dépendants par l'ensemble des seigneurs, mais de manière " non coordonnée ", c'est-à-dire interstitielle et invisible - et d'ailleurs sans que les seigneurs en soient eux-mêmes conscients, au-delà de la volonté et des motivations des agents dans leur recours à la faide. Lorsque le seigneur schirmt ses dépendants, c'est ainsi toujours des conséquences de sa propre action et réalisation en tant que seigneur qu'il le fait, sans pour autant qu'aucun des partenaires du rapport seigneurial n'en soit conscient.

5 L'examen, par la suite, de textes d'ordre narratif (chroniques) et idéologiques (traités sur les états de la société, pièces de théâtre pascales) est articulé d'une manière assez peu claire avec tout ce qui précède (et en particulier avec l'argument de la nonconscience ou de la non-explicité du double processus de soumission). Toutefois, l'analyse fine de ces textes par G.A. (auquel il faut cependant reprocher la très faible contextualisation desdits textes) fait clairement apparaitre que ces documents 1) ne s'adressent guère aux dépendants mais à la couche seigneuriale (cléricale, noble ou urbaine), pour laquelle les dépendants sont représentés comme des enjeux de concurrence (chaque seigneur devant s'approprier les dépendants des autres) ; surtout, 2) ils ne procèdent pas du tout à un démontage du système modélisé par G.A. mais à son habillage sous une autre logique implicite (la définition de deux catégories 
radicalement opposées), dramatisée à travers l'affirmation qu'il faut régulièrement piller les biens des " paysans " (le mot est là) pour éviter qu'ils ne deviennent arrogants. Cette " pédagogie sociale " contribue ainsi à désarticuler le système de domination en isolant la lutte entre seigneurs de la confrontation entre seigneurs et paysans.

6 L'ouvrage est tout à fait stimulant et exerce à certains endroits de la pratique historienne (notamment pour ce qui est de son rapport au langage, à la fois le langage scientifique/conceptuel et le langage des sources) des critiques ravageuses. Par ailleurs, on doit observer un certain déséquilibre pratique au sein de l'ouvrage : les aspects abstraits, conceptuels et modélisateurs sont mieux établis et développés que le traitement du corpus lui-même, ce qui risque de faire condamner d'autant plus facilement l'ouvrage par les adversaires de toute réflexion théorique que l'on pourra aligner des contre-exemples ou même critiquer tel ou tel aspect de pratique historienne. Mais surtout, ce qui pose le plus de problèmes est le schéma social sur lequel G.A. a bâti son analyse, à savoir le binôme seigneur/paysans. D'une part, la logique sociale de leur confrontation est réduite à un simple affrontement d'intérêts antagonistes, le seigneur n'apparaissant que comme une sorte de parasite, sans que soit prise en compte la nature particulière des rapports entre seigneurs et villageois : le seigneur contrôle l'accès au moyen de production (la terre et la force de travail), tandis qu'il est exclu du contrôle du procès de production (réalisé au niveau villageois); cette distorsion entre rapport et procès de production ne pouvait se résoudre que par la réaffirmation symbolique et pratique régulière de la domination seigneuriale, notamment par la faide (défendre ses dépendants, c'est réactualiser leur accès au moyen de production via le seigneur).

D'autre part, l'examen de la structure sociale des villages médiévaux du Sud-Ouest laisse apparaître (quand les sources le permettent...) face à la communauté d'habitants non pas un seigneur, mais un ensemble de seigneurs, dont certains dominaient parfois le même tenancier. Le principal problème de ces seigneurs, numériquement très inférieurs à la population dominée, était alors tout autant de dominer que d'assurer leur cohésion locale, ce à quoi servait, me semble-t-il, l'essentiel des " aveux de droits » : faire reconnaître par les dépendants eux-mêmes la répartition des pouvoirs (donc la cohésion seigneuriale) au niveau villageois. G.A. reconnaît certes l'existence de cette situation de concurrence (par exemple à l'arrière-plan de l'« aveu de droits " d'Ensheim, en 1435, qui ouvre son livre), mais il n'en tient aucun compte : chaque seigneur est seul face à ses paysans et seule la faide est propre à assurer à terme la reproduction $\mathrm{du}$ pouvoir seigneurial. L'isolement de ce binôme (G.A. utilise symptomatiquement le verbe herauspräparieren, qui sonne de manière biologique, comme une dissection et une mise en éprouvette) du continuum des rapports sociaux rend délicate l'appréciation de la portée des résultats, qui ne peuvent être valables que dans le cadre du protocole d'analyse. Il n'empêche qu'il s'agit là d'un livre stimulant et critique, dont on se prend à souhaiter qu'il y en ait davantage. 